\title{
Integrated films of transition metal oxides for information technology
}

\author{
Alexander A. Demkov ${ }^{1}$, Patrick Ponath ${ }^{1}$, Kurt Fredrickson ${ }^{1}$, Agham B. Posadas ${ }^{1}$, \\ Martin D. McDaniel ${ }^{2}$, Thong Q. Ngo ${ }^{2}$ and John G. Ekerdt ${ }^{2}$ \\ ${ }^{I}$ Department of Physics, The University of Texas at Austin, Austin, Texas, 78712, USA \\ ${ }^{2}$ Department of Chemical Engineering, The University of Texas at Austin, Austin, Texas, 78712, USA \\ E-mail address of corresponding author:emkov@physics.utexas.edu
}

\section{Abstract}

The recently developed ability to grow layers of transition metal oxides with atomic precision by means of physical vapor deposition has opened up a possibility of monolithic integration of these oxides on semiconductors. Here we review the recent progress in integrating ferroelectric films with $\mathrm{Si}$ and $\mathrm{Ge}$, and their potential applications in electronics and nanophotonics. Perovskite films described in the talk were grown by molecular beam epitaxy (MBE) and, when possible, chemical routes were tested via atomic layer deposition (ALD). Design of the structures and analysis of the experimental results were aided by density functional theory (DFT).

\section{Introduction}

Thin films of ferroelectric oxides grown directly on semiconductors have possible applications in non-volatile memory technology and nanophotonics. Nonvolatile semiconductor memories are essential in our daily lives, as largecapacity flash memories are used in solid state drives (SSD) of personal computers, mobile devices, digital cameras, moving-picture-expertsgroup (MPEG) audio players, universal-serial-bus (USB) memories, and other devices. Electrically erasable and programmable read-only memories (EEPROMs) and ferroelectric random access memories (FeRAMs) are used in wireless railway passes and IC (integrated circuit) or smart cards [1].

Among the new types of non-volatile memory, FeRAMs were the first to enter production. The most attractive feature of FeRAM is its low power consumption, which cannot be achieved by any other non-volatile memory. Because of this, FeRAMs have successfully competed even with flash memories, which require over $20 \mathrm{~V}$ for both write and erase operations. As FeRAM needs less than $2 \mathrm{~V}$, the voltage-pump-up circuit can be eliminated. Also, writing speed and the maximum number of rewrites of FeRAMs are, respectively, at least two and six orders of magnitude greater than those of flash memory. The main problem of this type of ferroelectric memory is that it uses a separate ferroelectric capacitor in addition to a transistor (see Fig. 1a), and thus is difficult to scale to high density.

A pure transistor-type ferroelectric memory cell has a structure similar to that of a MOS transistor but with the gate oxide replaced with a ferroelectric oxide (see Fig. 1b). Such a device would be competitive with DRAM in terms of memory bit density. However, at present, the ferroelectric films used in transistor-type ferroelectric memories have a thickness of about $100 \mathrm{~nm}$, which is too thick for gigabit-scale integration. Thus a thin ferroelectric film in monolithic integration with a conventional semiconductor is very attractive, particularly if long-term retention is not a critical issue such as in non-volatile caches for SSDs.

In addition, the large effective Pockels coefficient $r_{\text {eff }}=148 \mathrm{pmV}^{-1}$ (linear electro-optic effect) for high-quality epitaxial $\mathrm{BaTiO}_{3}$ (BTO) films on $\mathrm{Si}$ distinguishes $\mathrm{BTO}$ as a highly promising material for integrated silicon nanophotonics [2,3]. Since the first successful integration of perovskite oxides on $\mathrm{Si}$ [4] and the successive demonstration of ferroelectric functionality [5], electro-optic activity [2], and devices [3], it has been found that these properties are strongly dependent on the crystalline structure of ferroelectric BTO. In order to achieve a strong electro-optic response, the film must have proper orientation with respect to the applied electric field [6], and be able to sustain a strong electric field without breakdown [7].

Monolithic integration of BTO on $\mathrm{Si}$ is enabled by molecular beam epitaxy (MBE) through the use of buffer layers, including $\mathrm{BaO}[8], \mathrm{Ba}_{0.7} \mathrm{Sr}_{0.3} \mathrm{TiO}_{3}$ [9], and most commonly, $\mathrm{SrTiO}_{3}$ (STO) $[2,5,10]$. In what follows we shall describe our recent efforts to integrate ferroelectric BTO on $\mathrm{Si}$ and Ge using MBE and ALD.

\section{BTO on Si by MBE}

The epitaxial integration of $\mathrm{BaTiO}_{3}(\mathrm{BTO})$ on $\mathrm{Si}$ (001) presents several challenges, particularly for applications requiring that the ferroelectric polarization be pointing out of plane. The lattice mismatch between BTO and $\mathrm{Si}$ is $4 \%$ (and even larger at typical growth temperatures) with the BTO compressed in-plane. While the direction of the mismatch is favorable for producing $c$-axis oriented BTO, the relatively large mismatch usually results in a very high defect density that can degrade the electrical properties of the film. Further, there has not yet been any successful direct growth of BTO on $\mathrm{Si}$ as no suitable wetting interlayer that will enable direct epitaxy has been discovered. Another challenge is the large thermal expansion mismatch between BTO and Si, with BTO having a thermal expansion coefficient about three times larger than $\mathrm{Si}$. While BTO films have been grown on Si using various buffers [4,11-13], these typically produce $a$-axis oriented films as a result of the thermal expansion mismatch, which causes the BTO to experience tensile stress while cooling down 
through its Curie temperature. To resolve this problem, a combination of a suitable buffer that reduces both lattice and thermal expansion mismatch, and slow cool down to reduce stress is necessary to obtain $c$-axis oriented films on $\mathrm{Si}$.

Our strategy is to use a fully relaxed layer of STO grown on $\mathrm{Si}$ as a pseudosubstrate for the subsequent BTO deposition. Though initial attempts to grow epitaxial STO directly on Si were only partially successful [6], McKee and coworkers have introduced a growth process that resulted in device quality STO films [14]. The key of this approach is to use $1 / 2$ monolayer of $\mathrm{Sr}$ as a dual purpose template layer. First, the presence of $\mathrm{Sr}$ inhibits the oxidation of $\mathrm{Si}$ in a wide window of temperature and oxygen pressure. Second, the template results in wetting of Si by STO, enabling two dimensional growth [8]. The chemical mechanism of this effect is in the formation of an interfacial Zintl intermetallic that allows a low energy transition from the covalent bonding of Si to the ionic bonding of the oxide $[15,16]$. The lattice mismatch between BTO and relaxed STO still induces compressive strain that stabilizes out of plane orientation of the BTO film with a significantly reduced lattice mismatch of $2.2 \%$, as well as a reduced thermal expansion mismatch such that BTO now experiences favorable compressive stress during cool down (the BTO thermal expansion coefficient is slightly smaller than the STO thermal expansion coefficient). To further reduce the lattice mismatch between BTO and STO, we can also anneal the STO pseudosubstrate in oxygen prior to BTO deposition to allow for plastic relaxation of the $\mathrm{STO}$ on $\mathrm{Si}$ as a result of $\mathrm{SiO}_{\mathrm{x}}$ interlayer formation. We have shown that STO films on Si annealed in this manner result in an inplane lattice constant slightly larger than that of bulk STO [17]. By using this approach of tailoring the heterostructure layers and their interfaces, we show that we can overcome lattice and thermal expansion mismatch issues and grow $c$-axis oriented BTO films on Si with thicknesses up to 20 nm.

Lightly doped epi-grade $\mathrm{Si}(100)$ is cut into $20 \mathrm{x}$ $20 \mathrm{~mm}^{2}$ pieces and degreased using a sequence of acetone, isopropanol and deionized water for $5 \mathrm{~min}$ each with sonication. After drying, the substrate is then exposed to ultraviolet/ozone for $15 \mathrm{~min}$ to remove organic contaminants before being loaded into the MBE growth chamber. The Si substrate is then outgassed at $675^{\circ} \mathrm{C}$ for $10 \mathrm{~min}$ then dosed with 2 monolayers of $\mathrm{Sr}$ from an effusion cell followed by heating to $825^{\circ} \mathrm{C}$ to desorb the native silicon oxide using the so-called $\mathrm{Sr}$ deoxidation process [18]. After achieving a clear $2 \times 1$ surface reconstruction characteristic of a clean $\mathrm{Si}$ (100) surface as observed in reflection-high-energy electron diffraction (RHEED), the sample is cooled to $575^{\circ} \mathrm{C}$ and then dosed with additional $\mathrm{Sr}$ to form the Zintl phase template comprised of $1 / 2$ monolayer $\mathrm{Sr}$ (surface has $\mathrm{SrSi}_{2}$ stoichiometry) that is necessary for perovksite growth on $\mathrm{Si}(100)$. The characteristic RHEED pattern for the Zintl template layer is shown in Fig. 2(a). At moderate temperature, this Zintl phase template protects $\mathrm{Si}$ from oxidation and allows for nucleation and layer by layer growth of $\mathrm{SrTiO}_{3}$.

The Sr-templated Si substrate is cooled to $200^{\circ} \mathrm{C}$ and then molecular oxygen is allowed to flow into the DCA $600 \mathrm{MBE}$ chamber to a pressure of $8 \times 10^{-8}$ torr. A three-unit cell STO seed layer is first deposited at $200^{\circ} \mathrm{C}$ from $\mathrm{Sr}$ and $\mathrm{Ti}$ effusion cells calibrated to have the same flux $(\sim 1$ monolayer per min). The film is barely crystalline at this growth temperature. The sample is then heated in vacuum to $575^{\circ} \mathrm{C}$, causing the seed layer to fully crystallize [17]. Additional STO is deposited until a total thickness of $6 \mathrm{~nm}$ is reached at which STO is already fully relaxed to its bulk lattice constant. After the STO growth, the substrate is heated at $20^{\circ} \mathrm{C} / \mathrm{min}$ to $700^{\circ} \mathrm{C}$ while simultaneously ramping the $\mathrm{O}_{2}$ pressure to $5 \times 10^{-6}$ Torr. When the target $\mathrm{O}_{2}$ pressure and substrate temperature are reached, BTO growth by means of alternating monolayer dosing of $\mathrm{Ba}$ and $\mathrm{Ti}$ is initiated, with $\mathrm{Ba}$ first and $\mathrm{Ti}$ last. The entire growth process is monitored in situ by RHEED to ensure high crystallinity and stoichiometry. Fig. 2(b) shows a RHEED pattern after growth of a $20 \mathrm{~nm} \mathrm{BTO}$ film on STO/Si. For films thicker than $5 \mathrm{~nm}$, the growth was paused for $10 \mathrm{~min}$ after every $5 \mathrm{~nm}$ of growth. By doing these intermediate anneals, the high surface crystallinity is maintained throughout the entire growth process. After the desired thickness of BTO is grown, the sample is cooled at $5^{\circ} \mathrm{C} / \mathrm{min}$ in $\mathrm{O}_{2}$ to room temperature. After growth, x-ray diffraction and atomic force microscopy measurements are performed on the BTO films. An x-ray diffraction $2 \theta-\theta$ scan around the BTO (002) peak is shown in Fig. 3(a), and an atomic force microscope image of a BTO film after growth is shown in Fig. 3(b). Removed a sentence

\section{BTO on Ge by MBE}

For BTO growth on $\mathrm{Ge}(001)$ [19], the Ge surface is first prepared using a combination of wetetching, oxygen plasma cleaning and annealing, resulting in a $(2 \times 1)$ surface reconstruction, low surface roughness, and clear surface signal in the angle resolved photoemission spectrum [20]. Prior to BTO growth, $1 / 2$ monolayer of strontium is deposited to prevent germanium from oxidizing during the initial growth of the first 5 unit cells of $\mathrm{STO}$ at $200^{\circ} \mathrm{C}$. After increasing the temperature to $600^{\circ} \mathrm{C}$ to crystallize the STO, BTO is deposited on the STO/Ge template at a temperature of $650^{\circ} \mathrm{C}$.

Films were characterized by X-ray diffraction (XRD) performed on the BTO/Ge samples using a Philips X'Pert double-crystal diffractometer. Figure 4 shows symmetric $\theta-2 \theta$ scans of a $160-\AA$-thick 
BTO film grown on $\mathrm{Ge}$, both with and without a 20-Å-thick STO buffer layer. The BTO peaks correspond to a single orientation with an out-ofplane spacing of $4.06 \AA$ for the film with the STO buffer, and $3.98 \AA$ for the film without the buffer. This result clearly demonstrates the $c$-axis orientation for the STO-buffered sample compared with the $a$-axis orientation for the BTO layer grown directly on Ge. Rocking curve scans around the BTO 002 Bragg peak typically show a full-width at half-maximum of $0.5-0.8^{\circ}$.

The ferroelectric properties of the BTO layer were tested using BE-piezoresponse spectroscopy measurements (BEPS [21]). We performed a BEPS measurement on the BTO film across a $10 \times 10$ grid to yield a data set of 100 measurements in total. Three selected responses at individual points from the 100 measurements, after fitting to a simple harmonic oscillator model, are shown in Fig.5(b,c). The average of 100 measurements is shown as a solid olive line. The amplitude curves clearly follow the expected butterfly-like hysteresis typical of ferroelectrics, while the phase loop appears to show a change somewhat $<180^{\circ}$.

\section{STO on Si and Ge by ALD}

Crystalline STO films have been grown by ALD on $\mathrm{Si}(001)$ substrates using a four-unit-cell STO template layer grown by MBE [22]. Stoichiometric, crystalline films were obtained using a 1:1 (Sr:Ti) cycle ratio of strontium bis(triisopropylcylopentadienyl) and titanium tetraisopropoxide, with a water co-reactant, at a deposition temperature of $250{ }^{\circ} \mathrm{C}$. XRD and crosssectional TEM analysis verified the high degree of crystallinity in the as-deposited STO films. In situ XPS analysis revealed minimal, if any, interfacial amorphous layer at the STO-Si interface. The successful integration of epitaxial oxide films on Si(001) substrates by ALD, using a thin STO buffer layer grown by MBE, is a promising method for large-scale fabrication of multifunctional perovskites integrated with semiconductor technology [23].

We have also grown crystalline STO directly on germanium via a purely chemical method, atomic layer deposition (ALD). In situ X-ray photoelectron spectroscopy confirms the presence of stoichiometric STO with no $\mathrm{GeO}_{\mathrm{x}}$ formation or carbon impurities observed [24]. Epitaxial STO films up to $15-\mathrm{nm}$ thick with a high-degree of crystallinity were grown on the Ge (001) substrate. The crystalline structure and orientation are confirmed via electron and x-ray diffraction. Capacitance-voltage and current-voltage measurements were performed on a $15-\mathrm{nm}$ thick undoped STO film. The undoped STO showed a large dielectric constant of $\sim 90$; however, the leakage current was unacceptably high $\left(\sim 10 \mathrm{~A} / \mathrm{cm}^{2}\right.$ at $0.7 \mathrm{MV} / \mathrm{cm})$. To lower the leakage current, the STO films were doped with aluminum since it is known to increase the band gap of STO [25]. An 8$\mathrm{nm}$ thick Al-doped STO film showed a leakage current density $\sim 0.1 \mathrm{~A} / \mathrm{cm}^{2}$ at $0.7 \mathrm{MV} / \mathrm{cm}$, roughly two orders of magnitude lower than the undoped STO.

\section{BTO on Si by ALD}

Ngo et al. have grown $c$-axis oriented epitaxial BTO at $225{ }^{\circ} \mathrm{C}$ using ALD with barium bis(triisopropylcyclopentadienyl) on 1.6-nm STObuffered $\mathrm{Si}(001)$ [26]. The BTO films have a good degree of crystallinity and no amorphous layer was observed at the $\mathrm{STO} / \mathrm{Si}$ interface. A 5-min vaccum anneal at $600{ }^{\circ} \mathrm{C}$ significantly improved BTO crystallinity. Annealing using a relatively high temperature ramping rate $\left(30^{\circ} \mathrm{C} / \mathrm{min}\right)$ could destroy the tetragonal structure, while annealing using a lower ramping rate $\left(5^{\circ} \mathrm{C} / \mathrm{min}\right)$ is able to maintain the $c$-axis oriented tetragonal structure. The dielectric constant of the BTO/STO stack on Si was estimated to be $\sim 660$ from capacitance-voltage measurements. We expect the ALD method of growing $c$-axis oriented epitaxial BTO on STObuffered $\mathrm{Si}(001)$ at low temperature to be a potential way to fabricate the negative capacitance gate oxide structure on $\mathrm{Si}$ with no amorphous layer between STO and Si in the near future.

\section{Density functional theory}

Theory has played a very important role in the development of this field. We owe to DFT our understanding of the perovskite/diamond-type interface beginning with the knowledge of its atomic structure [27-32]. The fundamental role of the Zintl template to prevent the oxidation of Si has been elucidated through first principles calculations [33-45]. The exact atomic positions of the interfacial Zintl layer are crucial for determining the correct band offsets of the interface. For example, the $\mathrm{BTO} / \mathrm{Ge}$ interface is strongly controlled by the formation of $\mathrm{a} \mathrm{SrO}_{2}$ interfacial dipole, which influences the charge transfer from Ge to BTO. The change in the value of this dipole due to atomic position can change the valence band offsets by as much as $3 \mathrm{eV}$. Although electron microscopy gives some information of the interfacial structure, it is extremely difficult to pick up atomic positions for lighter elements such as $\mathrm{O}$, which must be located through the aid of theoretical investigation. Theory is an important tool to reveal the many possible interfaces, and is used to compute physical properties that can be compared with experiment to correctly identify the interface.

Theory has also demonstrated the ability of the ferroelectric oxide to modulate charges in the underlying semiconductor layer even with a thin high-k insulator in between [19]. Crucially, DFT has shown that the charge modulation is not localized in the interfacial STO on Ge as it does for $\mathrm{SiO}_{2}$ on $\mathrm{Si}$, and the charge modulation can be seen in $\mathrm{Ge}$ as well, which is necessary for the 
ferroelectric field effect (Figure 6). This work is an important foundation for the creation of ferroelectric devices with strong support from theoretical calculations.

\section{Conclusion}

We have only just begun to scratch the surface of the possibilities for the integration of crystalline ferroelectric materials on $\mathrm{Si}$ and Ge. The use of a robust transition layer for $\mathrm{Si}(001)$ coupled with growth methods that allow for management of ferroelectric strain and orientation, as well as the degree of coupling to $\mathrm{Si}$ affords many device possibilities on existing Si platforms. The ability to grow ferroelectric materials directly on $\mathrm{Ge}(001)$ without a transition layer and without the possible formation of an insulating low-k interfacial layer presents new opportunities as the industry moves from silicon to germanium platforms. Further research will undoubtedly increase both the number of ferroelectric materials integrated with semiconductors and the variety of functional devices that can be fabricated using these materials, along with processes that allow the effective creation of these devices. It is clear that both theory and experiment must be used in complementary fashion in order to complete the development of fully integrated ferroelectric devices.

\section{Acknowledgements}

We wish to thank C. Dubourdieu, S. Kalinin, J. Bruley, D. J. Smith and K. J. Lai without whose support and collaboration this research would not be possible. And we thank K. J. Kormondy for critically reading the manuscript. The work was supported by the Air Force Office of Scientific Research (FA9550-12-10494) and the National Science Foundation (Award DMR-1207342).

\section{References}

[1] Y. Fujisaki, Jpn. J. Appl. Phys. 52 (2013) 040001.

[2] S. Abel, T. Stöferle, C. Marchiori, C. Rossel, M.D. Rossell, R. Erni, D. Caimi, M. Sousa, A. Chelnokov, B.J. Offrein, J. Fompeyrine, Nat. Commun. 4 (2013) 1671.

[3] C. Xiong, W.H.P. Pernice, J.H. Ngai, J.W. Reiner, D. Kumah, F.J. Walker, C.H. Ahn, H.X. Tang, Nano Lett. 14 (2014) 1419.

[4] R.A. McKee, F.J. Walker, J.R. Conner, E.D. Specht, D.E. Zelmon, Appl. Phys. Lett. 59 (1991) 782.

[5] C. Dubourdieu, J. Bruley, T.M. Arruda, A. Posadas, J. Jordan-Sweet, M.M. Frank, E. Cartier, D.J. Frank, S. V Kalinin, A.A. Demkov, V. Narayanan, Nat. Nanotechnol. 8 (2013) 748.
[6] B.W. Wessels, Annu. Rev. Mater. Res. 37 (2007) 659.

[7] R. Pan, Y. He, M. Li, P. Li, P. Liu, Z. Xia, Mater. Sci. Eng. B 188 (2014) 84.

[8] R.A. McKee, F.J. Walker, M.F. Chisholm, Science 293 (2001) 468.

[9] V. Vaithyanathan, J. Lettieri, W. Tian, A. Sharan, A. Vasudevarao, Y.L. Li, A. Kochhar, H. Ma, J. Levy, P. Zschack, J.C. Woicik, L.Q. Chen, V. Gopalan, D.G. Schlom, J. Appl. Phys. 100 (2006) 024108.

[10] F. Niu, B.W. Wessels, J. Vac. Sci. Technol. B Microelectron. Nanom. Struct. 25 (2007) 1053.

[11] M.-B. Lee, M. Kawasaki, M. Yoshimoto, H. Koinuma, Appl. Phys. Lett. 66 (1995) 1331.

[12] Z. Yu, J. Ramdani, J.A. Curless, C.D. Overgaard, J.M. Finder, R. Droopad, K.W. Eisenbeiser, J.A. Hallmark, W.J. Ooms, V.S. Kaushik, J. Vac. Sci. Technol. 18 (2000) 2139.

[13] A.R. Meier, F. Niu, B.W. Wessels, J. Cryst. Growth 294 (2006) 401.

[14] R.A. McKee, F.J. Walker, M.F. Chisholm, Phys. Rev. Lett. 81 (1998) 3014.

[15] A.A. Demkov, H. Seo, X. Zhang, J. Ramdani, Appl. Phys. Lett. 100 (2012) 071602.

[16] X. Zhang, A. Demkov, H. Li, X. Hu, Y. Wei, J. Kulik, Phys. Rev. B 68 (2003) 125323.

[17] M. Choi, A. Posadas, R. Dargis, C.-K. Shih, A. a. Demkov, D.H. Triyoso, N. David Theodore, C. Dubourdieu, J. Bruley, J. Jordan-Sweet, J. Appl. Phys. 111 (2012) 064112.

[18] Y. Wei, X. Hu, Y. Liang, D.C. Jordan, B. Craigo, R. Droopad, Z. Yu, A. Demkov, J.L. Edwards, W.J. Ooms, J. Vac. Sci. Technol. B 20 (2002) 1402.

[19] P. Ponath, K. Fredrickson, A.B. Posadas, Y. Ren, X. Wu, R.K. Vasudevan, M. Baris Okatan, S. Jesse, T. Aoki, M.R. McCartney, D.J. Smith, S. V. Kalinin, K. Lai, A.A. Demkov, Nat. Commun. 6 (2015) 6067.

[20] P. Ponath, A.B. Posadas, R.C. Hatch, A. a. Demkov, J. Vac. Sci. Technol. B 31 (2013) 031201.

[21] S. Jesse, A.P. Baddorf, S. V. Kalinin, Appl. Phys. Lett. 88 (2006) 062908. 
[22] M.D. McDaniel, A. Posadas, T.Q. Ngo, A. Dhamdhere, D.J. Smith, A.A. Demkov, J.G. Ekerdt, J. Vac. Sci. Technol. A 31 (2013) 01A136.

[23] D.J. Frank, P.M. Solomon, C. Dubourdieu, M.M. Frank, V. Narayanan, and T.N. Theis, IEEE Trans. Electron Dev. 61 (2014) 2145.

[24] M.D. McDaniel, T.Q. Ngo, A. Posadas, C. Hu, S. Lu, D.J. Smith, E.T. Yu, A.A. Demkov, J.G. Ekerdt, Adv. Mater. Interfaces 1 (2014) 1400081.

[25] A. B. Posadas, C. Lin, A. A. Demkov and S. Zollner, Appl. Phys. Lett. 103, 142906 (2013).

[26] T.Q. Ngo, A.B. Posadas, M.D. McDaniel, C. Hu, J. Bruley, E.T. Yu, A.A. Demkov, J.G. Ekerdt, Appl. Phys. Lett. 104 (2014) 082910.

[27] K.D. Fredrickson, P. Ponath, A.B. Posadas, M.R. McCartney, T. Aoki, D.J. Smith, A.A. Demkov, Appl. Phys. Lett. 104, (2014) 242908

[28] X. Zhang, A.A. Demkov, H. Li, X. Hu, Y. Wei, J. Kulik, Phys. Rev. B 68, (2003) 125323

[29] C.R. Ashman, C.J. Foerst, K. Schwarz, P.E Bloechl, Phys. Rev. B 69, (2004) 075309

[30] A.M. Kolpak, S. Ismail-Beigi, Phys. Rev. B 85, (2012) 195318

[31] A.M. Kolpak, F.J. Walker, J.W. Reiner, Y. Segal, D. Su, M.S. Sawicki, C.C. Broadbridge, Z. Zhang, Y. Zhu, C.H. Ahn, S. Ismail-Beigi, Phys. Rev. Lett. 105, (2010) 217601

[32] C.S. Hellberg, K.E. Anderson, H. Li, P.J. Ryan, J.C. Woicik, Phys. Rev. Lett. 108, (2012) 166101

[33] K.D. Fredrickson, H. Seo, A.A. Demkov, in preparation

[34] H. Watanabe, K. Kato, T. Uda, K. Fujita, M Ichikawa, T. Kawamura, K. Terakura, Phys. Rev. Lett. 80, (1998) 345

[35] H. Kageshima, K. Shiraishi, Phys. Rev. Lett. 81, (1998) 26

[36] T. Uchiyama, M. Tsukada, Phys. Rev. B 55, (1997) 9356

[37] T. Uchiyama, M. Tsukada, Phys. Rev. B. Condens. Matter 53, (1996) 7917

[38] C.H. Choi, D.-J. Liu, J.W. Evans, M.S. Gordon, J. Am. Chem. Soc. 124, (2002) 8730
[39] F. Fuchs, W.G. Schmidt, F. Bechstedt, J. Phys. Chem. B 109, (2005) 17649

[40] C. Mastail, I. Bourennane, A. Estève, G. Landa, M.D. Rouhani, N. Richard, A. Hémeryck, IOP Conf. Ser. Mater. Sci. Eng. 41, (2012) 012007

[41] A. Hemeryck, N. Richard, A. Estève, M.D. Rouhani, J. Non. Cryst. Solids 353, (2007) 594

[42] Y. Miyamoto, A. Oshiyama, Phys. Rev. B 43, (1991) 9287

[43] K. Kato, T. Uda, Phys. Rev. B 62, (2000) 15978

[44] K. Kato, T. Uda, K. Terakura, Phys. Rev. Lett. 80, (1998) 2000

[45] A.A. Demkov, O.F. Sankey, Phys. Rev. Lett. 83, (1999) 10 
a)

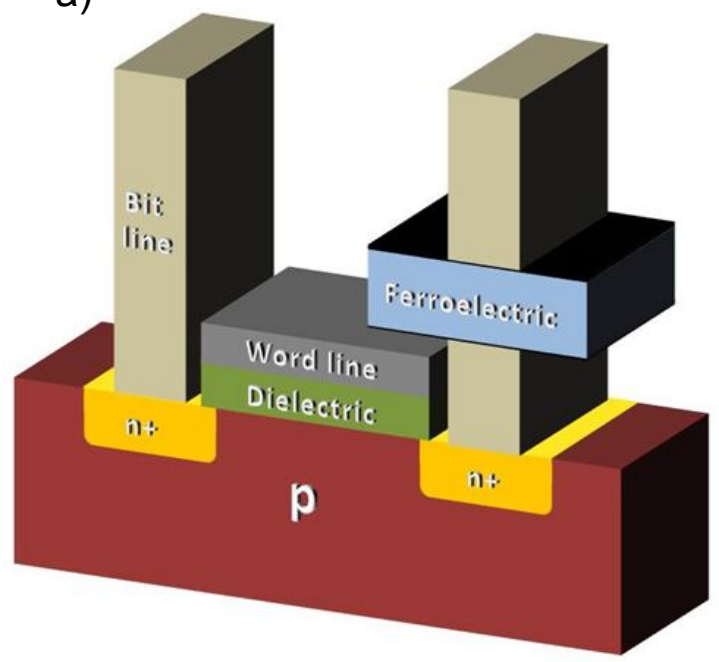

b)

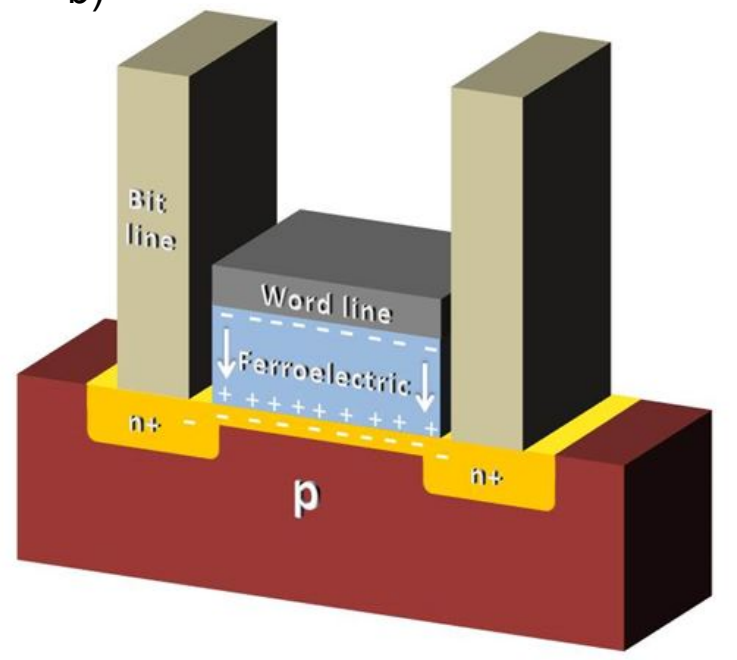

Figure 1: a) The structure of a 1T-1C FeRAM and b) a transistor-type FeRAM. In a) a ferroelectric material replaces the dielectric of the capacitor, while in b) it replaces the dielectric of the transistor.
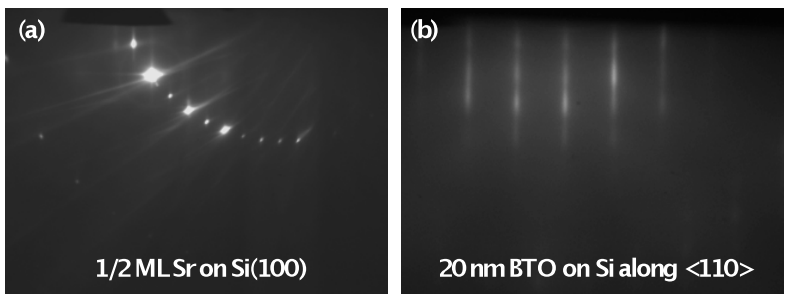

Figure 2: RHEED pattern for (a) Sr Zintl template on $\mathrm{Si}$ along $\mathrm{Si}<110>$ showing a sharp $2 \mathrm{x}$ reconstruction pattern with an atomically flat surface; (b) $20 \mathrm{~nm} \mathrm{BTO}$ grown on $\mathrm{STO} / \mathrm{Si}$ along the $<110>$ azimuth showing a streaky $1 \mathrm{x}$ pattern.
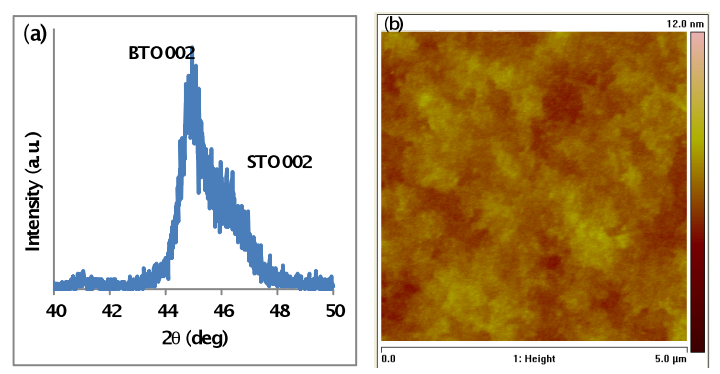

Figure 3: (a) X-ray diffraction pattern of $10 \mathrm{~nm}$ BTO on $6 \mathrm{~nm}$ STO on $\mathrm{Si}$ showing c-axis orientation of BTO. (b) Atomic force microscopy (AFM) of stoichiometric BTO film grown on $\mathrm{STO} / \mathrm{Si}$ over a $5 \times 5 \mu \mathrm{m}^{2}$ area with a root mean square roughness of $\sim 0.5 \mathrm{~nm}$. 


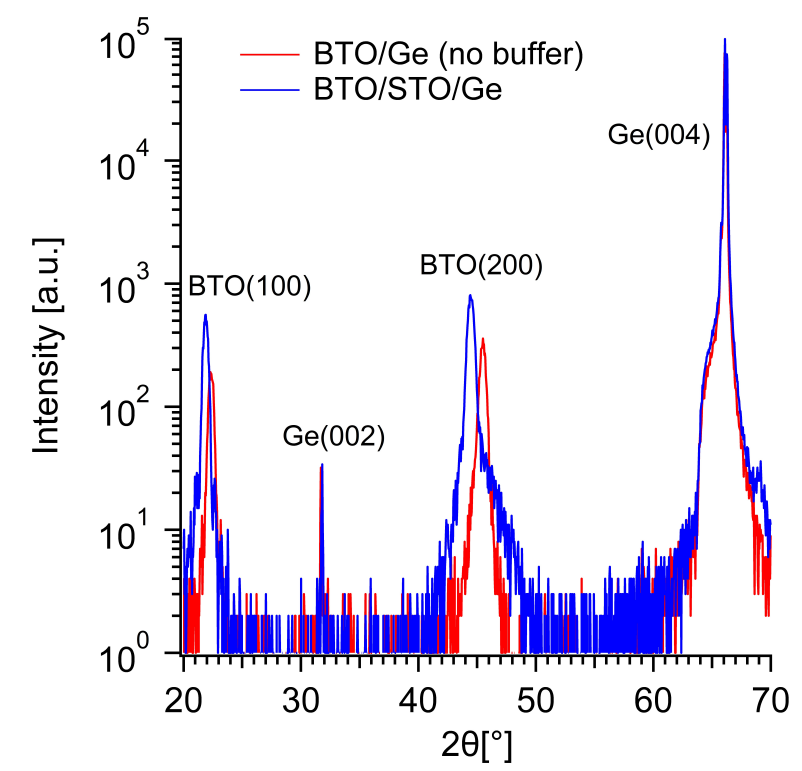

Figure 4: X-ray diffraction $2 \theta-\theta$ scan of $16 \mathrm{~nm} \mathrm{BaTiO}{ }_{3}$ epitaxially grown on $\mathrm{Ge}(001)$ substrate, with and without a $2 \mathrm{~nm} \mathrm{SrTiO}$ buffer layer. Without the buffer, the d-spacing corresponds to the $a$ axis of BTO directed out of plane. With the STO buffer, the $c$ axis of BTO is directed out of plane. Figure taken from [19]. 

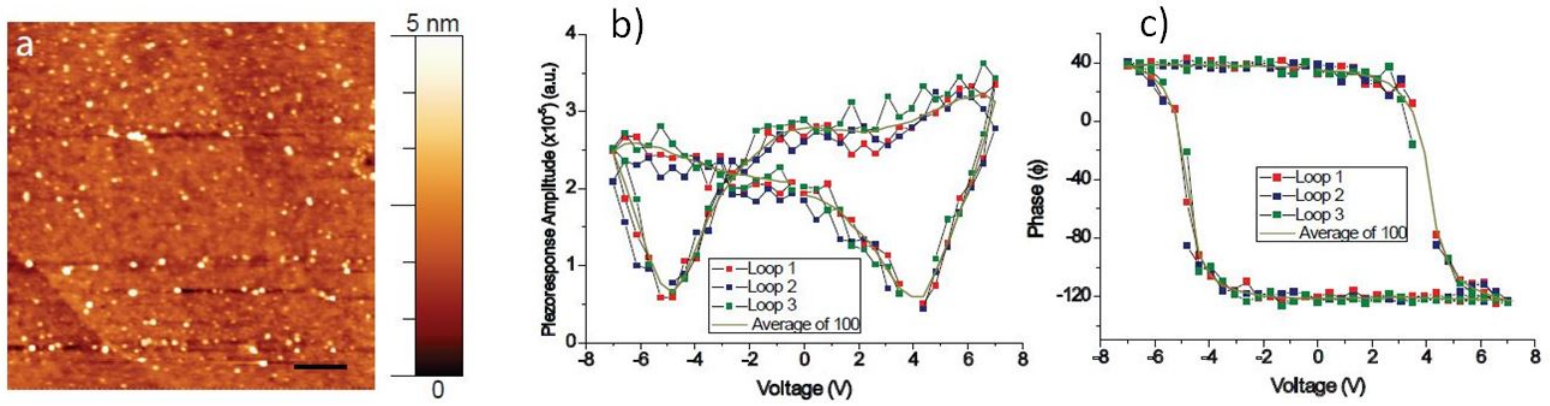

Figure 5: (a) Topography. Hundred hysteresis loops were captured using BE spectroscopy. Selected amplitude and phase loops are shown in (b) and (c), respectively, with the average loop shown as a solid line in olive, showing ferroelectric behavior. Figure taken from [19]. 


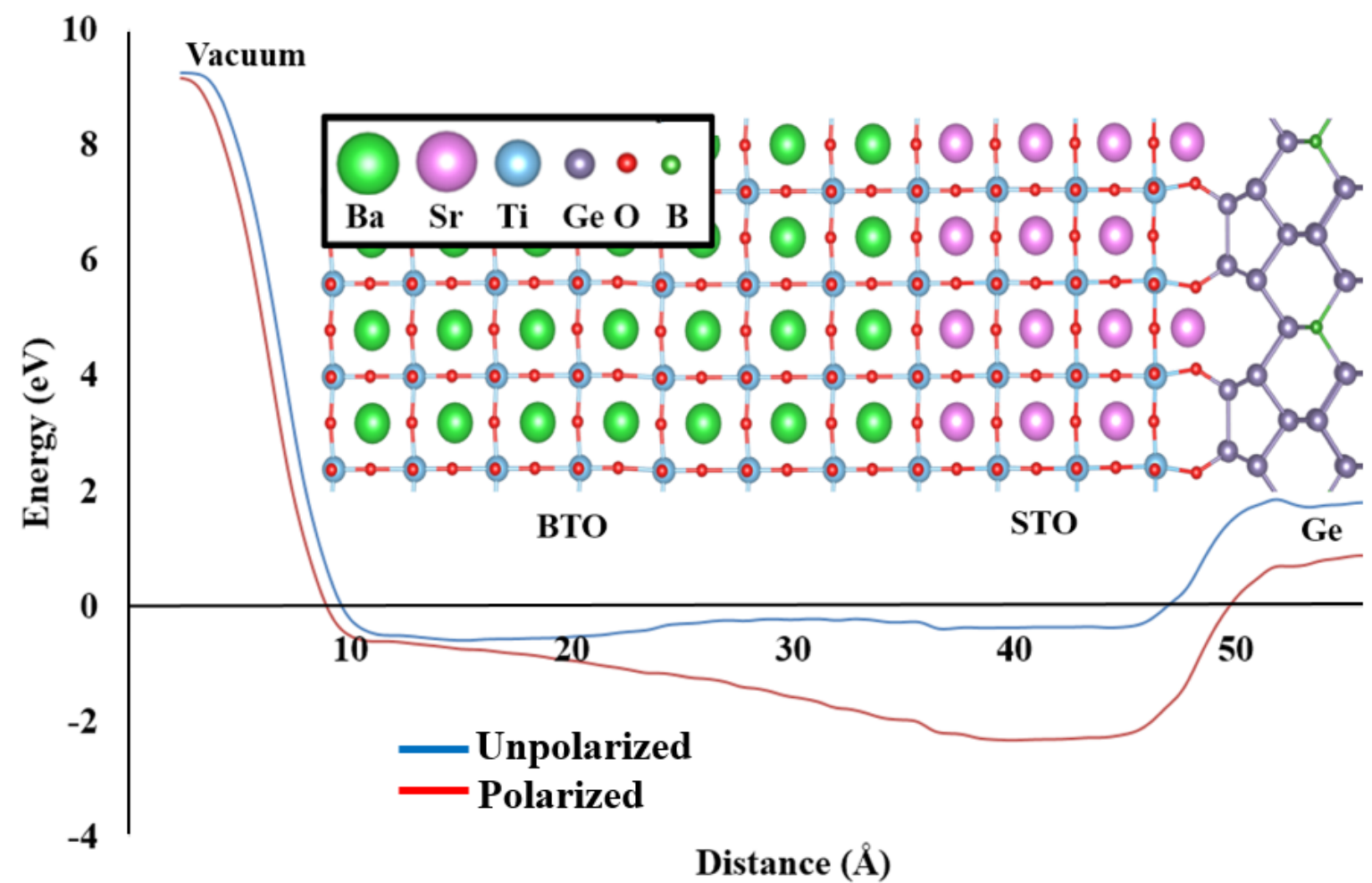

Figure 6: The simulation cell for the $\mathrm{BTO} / \mathrm{STO} / \mathrm{Ge}$ structure; Ge is p-type (B doped). Below is the planeaveraged electrostatic potential, showing the change in potential in Ge depending on the polarization state. 


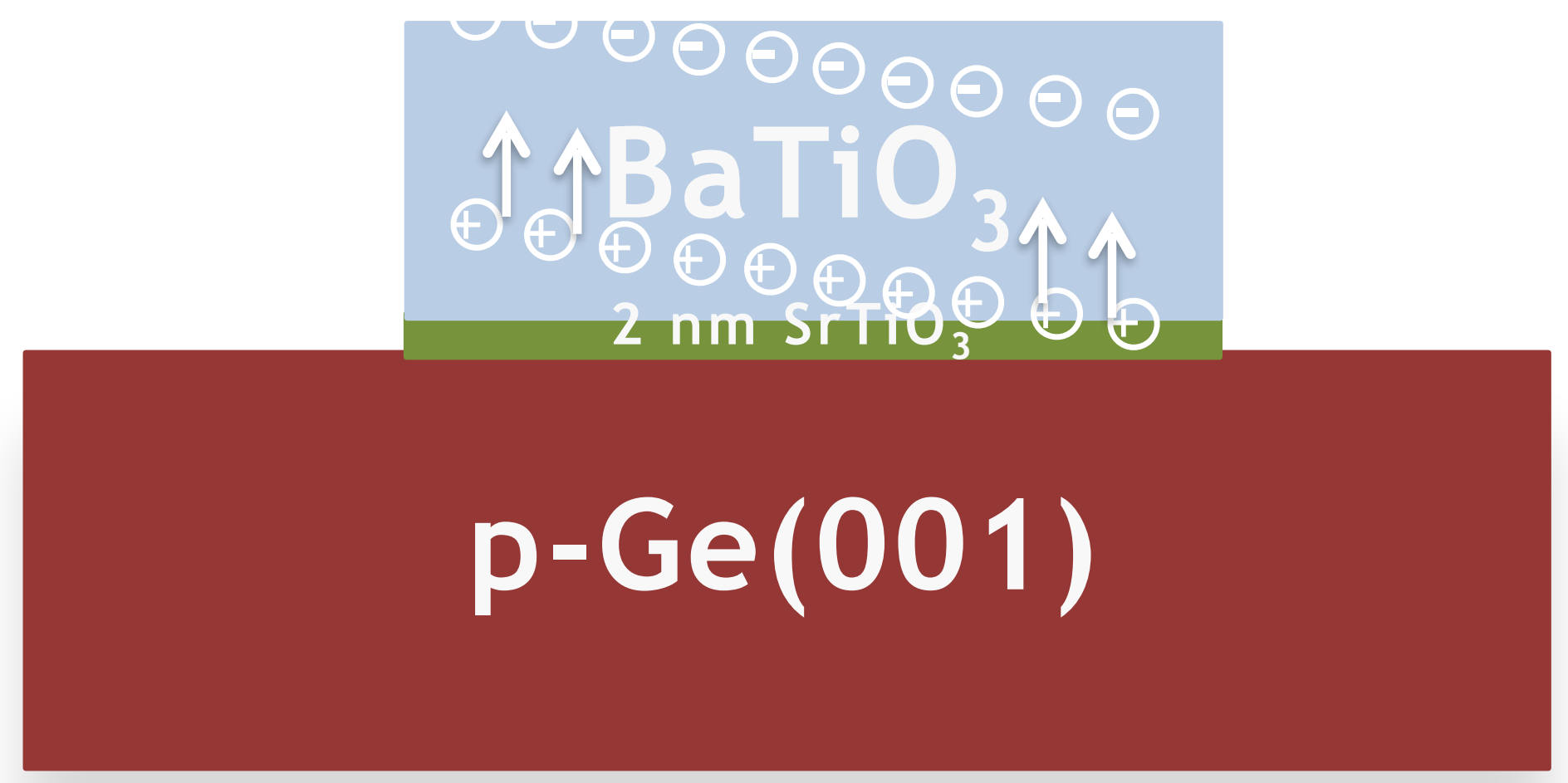

